\title{
Cholecystolithiasis mimicking pyloric stenosis
}

\author{
${ }^{*}$ Ramesh Y Bhat ${ }^{1}$, Sandesh Kini' ${ }^{2}$ \\ Sri Lanka Journal of Child Health, 2021; 50(4): 731-732 \\ DOI: http://doi.org/10.4038/sljch.v50i4.9901 \\ (Key words: Persistent vomiting, Neonate, Pyloric stenosis, Cholecystolithiasis)
}

\begin{abstract}
Introduction
Causes of persistent vomiting in an otherwise healthy neonate or young infant include faulty feeding, aerophagia, congenital pyloric stenosis, severe gastro-esophageal reflux disease or upper gastrointestinal obstruction ${ }^{1-4}$. The advice of correct feeding technique and proper burping after feeding usually resolves the minor ailment. Projectile nonbilious vomiting and upper abdominal distension with a palpable mass suggest the diagnosis of congenital hypertrophic pyloric stenosis (CHPS). Ultrasonography further confirms the diagnosis. Authors report a one-month-old infant who was referred as a case of CHPS because of persistent severe vomiting, abdominal distension and poor feeding. The abdominal ultrasonography findings revealed an entirely different rare entity, cholecystolithiasis. The treatment, course in the hospital and follow up of the case is discussed.
\end{abstract}

\section{Case report}

A one-month-old infant was referred to our hospital for persistent vomiting, poor feeding and upper abdominal distension for the past 5 days with a suspicion of CHPS. There was a history of fever and weight loss. The infant used to vomit after every feed that was given. He was born at term gestation by caesarean section. He cried soon after birth, had a birth weight of $3200 \mathrm{~g}$ and had no postnatal complications. The past history revealed infrequent vomiting episodes in the preceding two weeks.

Examination revealed a weight of $4600 \mathrm{~g}$, a length of $53.5 \mathrm{~cm}$ and a head circumference of $38 \mathrm{~cm}$. Vital functions were normal and there was no jaundice.

1Professor and Unit Head, Department of
Paediatrics, ${ }^{2}$ Associate Professor, Kasturba
Medical College, Manipal Academy of Higher
Education (MAHE) University, Manipal, India
*Correspondence: docramehbhat@yahoo.co.in

https://orcid.org/0000-0003-2919-6361

(Received on 18 November 2020: Accepted after revision on 18 December 2020)

The authors declare that there are no conflicts of interest.

Personal funding was used for the project.

Open Access Article published under the Creative Commons Attribution CC-BY (C) (i) License
Abdomen was distended, there was no visible gastric peristalsis and no definite mass was identified. Cardiorespiratory system and central nervous system examination were normal.

Laboratory investigations revealed a haemoglobin (Hb) level of $9.7 \mathrm{~g} \%$, total leucocyte count of $6200 / \mathrm{mm}^{3}$ (neutrophils $16 \%$, lymphocytes $60.9 \%$ ) and a platelet count of $587,000 / \mathrm{mm}^{3}$. Alanine aminotransferase level was $30 \mathrm{IU} / \mathrm{L}$, aspartate aminotransferase level was 63IU/L, alkaline phosphatase level was $487 \mathrm{U} / \mathrm{L}$, total bilirubin was $0.31 \mathrm{mg} / \mathrm{dL}$ and serum creatinine was $0.2 \mathrm{mg} / \mathrm{dL}$. Reticulocyte count was $1.3 \%$. Direct Coombs test was negative. Mean corpuscular hemoglobin concentration was $34.2 \mathrm{~g} / \mathrm{dL}$. Smear showed no evidence of haemolysis or sickle cells. Urine was negative for bile salt or bile pigments.

A diagnosis of pyloric stenosis or gastroesophageal reflux was suspected. Ultrasonography of abdomen was performed and it showed distended gall bladder with calculus measuring $3.7 \times 2.3 \mathrm{~cm}$. There was a pericholecystic fluid collection. The pyloric muscle thickness and the canal length were normal. There were no other abnormalities detected in the abdomen. The diagnosis of cholecystolithiasis with infection was considered. Because of fever, intravenous (IV) antibiotics were given. As the baby did not tolerate any feed orally, IV fluids were given for 24 hours. Ursodeoxycholic acid was added. By 72 hours, the vomiting decreased and baby started breastfeeding well. He was discharged after 5 days of hospitalization as the vomiting completely subsided. At 4 weeks follow up, the infant was asymptomatic, gained weight and ultrasonography suggested decreasing size of the gall stone.

\section{Discussion}

A young infant presented with severe persistent vomiting mimicking CHPS but ultrasonography findings confirmed the diagnosis of cholecystolithiasis with infection. Cholelithiasis is an extremely unusual finding in infancy ${ }^{1-3}$. In patients less than 18 years old with gallstones, only $15 \%$ occurs in infancy ${ }^{4}$. The present case is just a one month old infant.

CHPS is a common surgical cause of infantile vomiting. Infant can present with failure to retain feeds, persistent non-bilious vomiting following 
feeds, a palpable epigastric mass and dehydration. In a suspected CHPS, ultrasound is the imaging modality of choice to reliably establish the diagnosis. The sonographic parameters such as pyloric length, pyloric diameter, muscle thickness and pyloric volume help to arrive at the diagnosis. In the present case all these parameters were normal.

The risk factors associated with developing gallstones in infancy include prematurity, haemolytic conditions, ileal resection, sepsis, total parenteral nutrition and post cardiac surgery ${ }^{3,4}$. Only $20 \%$ of gallstones in infants and children are due to haemolytic disease. Most gallstones in infants and young children are composed primarily of calcium bilirubinate pigment with varying quantities of cholesterol and calcium carbonate. Symptoms include a right upper quadrant mass, emesis, jaundice and acholic stools ${ }^{3}$. The present case had severe emesis, aetiology was nonhaemolytic and there were no risk factors. Jeanty $\mathrm{C}$, et $a l^{3}$ reported that 5 out of 50 infants studied over a 16-year period did not have any risk factors.

Outcome of infantile cholelithiasis ranges from spontaneous resolution to choledocholithiasis or cholecystitis. Management includes antibiotics, ursodeoxycholic acid (UDCA), elective cholecystectomy, emergency operative exploration or observation. The spontaneous resolution rate of infantile cholelithiasis is about $35-60 \% 0^{3,4}$. Bogue $\mathrm{CO}$, et $a l^{4}$ reported spontaneous resolution of gall stones in approximately one third of cases at an average of 9 months after initial diagnosis ${ }^{4}$. Jeanty $\mathrm{C}$, et $a l^{3}$ managed 38 of 50 cholelithiasis conservatively, 20 among them having serial ultrasound examinations and one fourth had resolution of their gallstones at a median age of 12.2 (IQR 1.9-18.5) months following diagnosis. With high anaesthetic and surgical risks in infancy, initial conservative management is the treatment of choice in selected patients. In the present case conservative management with antibiotics, UDCA and supportive care resulted in resolution of the gall stone.
Severe persistent vomiting in young infants may have various underlying conditions that may mimic one another. Abdominal ultrasonography helps to identify or rule out CHPS when the symptoms are suggestive of it. Further, ultrasonography can also identify other rare conditions like cholecystolithiasis in young infants. Cholecystolithiasis in young infants is uncommon; however, prompt diagnosis and conservative management helps in decreasing the morbidity

\section{References}

1. Asabe K, Handa N. Infant cholelithiasis: Report of a case. Surgery Today 1997; 27: 71-5.

https://doi.org/10.1007/BF01366944

PMid: 9035305

2. Holcomb GW Jr, Holcomb GW. Cholelithiasis in infants, children, and adolescents. Pediatrics in Review 1990; 11; 268-74.

https://doi.org/10.1542/pir.11-9-268

PMid: 2408028

3. Jeanty C, Derderian SC, Courtier J, Hirose S. Clinical management of infantile cholelithiasis. Journal of Pediatric Surgery 2015; 50:1289-92. https://doi.org/10.1016/j.jpedsurg.2014.10 .051

PMid: 25783306

4. Bogue CO, Murphy AJ, Gerstle JT, et al. Risk factors, complications, and outcomes of gallstones in children: a single-centre review. Journal of Pediatric Gastroenterology and Nutrition 2010; 50: 303-8. https://doi.org/10.1097/MPG.0b013e3181 b99c72

PMid: 20118803 